RESIDENT

\& FELLOW

SECTION

Section Editor

Mitchell S.V. Elkind,

MD, MS

Michelle L. Mauermann, MD

Ted M. Burns, MD

Address correspondence and reprint requests to Dr. Michelle

L. Mauermann, Mayo Clinic, 200

First Street SW., Rochester, MN

55902

mauermann.michelle@mayo.edu

\title{
Pearls and Oy-sters: Evaluation of peripheral neuropathies
}

Peripheral neuropathy is common, with an estimated prevalence of $\sim 2 \%$ in the general population and prevalence as high as $8 \%$ in those over the age of 55 years. ${ }^{1}$ Residents and fellows will undoubtedly evaluate many patients with various types of peripheral neuropathies during their careers. The evaluation for the etiology of a patient's peripheral neuropathy can be challenging given the vast number of potential etiologies ${ }^{2,3}$ and the fact that despite an appropriate evaluation, a high percentage of cases will remain idiopathic. ${ }^{4,5}$ In this article, we provide a simple, easy-toremember algorithm that can simplify the evaluation of peripheral neuropathy.

PERIPHERAL NEUROPATHY ALGORITHM The evaluation of peripheral neuropathy should begin with the gathering of clinical data including the history of present illness, past medical history, family history, and physical examination. The determination of what of that clinical data is relevant requires knowledge of various diseases and the risk factors for those diseases. The information then needs to be synthesized in order to characterize the complaints and findings and determine an appropriate workup. The appropriate characterization of the complaints and findings is essential in order to perform an efficient, cost-effective, and successful evaluation. We recommend characterizing the neuropathy by answering four basic questions about the neuropathy: What? Where? When? What setting? (figure). ${ }^{6}$ This simple algorithm should facilitate the early step in clinical reasoning of creating a mental abstraction-that is, a problem representation or one-sentence summary of the defining and discriminating characteristics of the case. ${ }^{7}$ The creation of an accurate problem representation will allow the evaluating physician to focus on a small list of potential etiologies. This critical step in diagnostic reasoning will usually guide the physician down the correct diagnostic path, but it must be kept in mind that our algorithm is not absolute and there will always be cases that prove to be exceptions. The creation and utilization of problem representations will also serve as anchor points in memory that should allow the physician to more efficiently evaluate future cases and particularly to identify subtle but meaningful variations in similar cases. ${ }^{7}$

PEARLS FOR EVALUATING PERIPHERAL NEUROPATHY What? Determine what nerve fiber modalities-motor, sensory, or autonomic — are involved. If sensory fibers are involved, sensory symptoms should be characterized into those with a positive sensation, such as prickling, tingling, or buzzing, vs those with a negative sensation, such as loss of sensation or imbalance. This differentiation is helpful because these positive sensory symptoms often suggest a neuropathy is acquired rather than inherited. ${ }^{4}$ Acquired neuropathies with large fiber dysfunction (either motor or sensory with loss of vibration, proprioception, or light touch) due to demyelination often have symptoms of weakness with or without ataxia with positive sensory symptoms such as tingling. Those with small fiber dysfunction (loss of pain or temperature) often have symptoms such as burning without weakness or ataxia. Small fiber neuropathies can have positive sensory symptoms such as burning, regardless of whether they are acquired or inherited. However, most types of inherited neuropathies tend to have more signs (examination abnormalities) than symptoms. The identification of sensory nerve fiber involvement in almost all instances excludes disorders of the anterior horn cell, neuromuscular junction, and muscle. Deciding whether weakness or sensory symptoms are more prominent can also be helpful because the inflammatory demyelinating neuropathies often manifest with weakness overshadowing sensory nerve fiber disturbance. Autonomic nerve involvement can be an important clue because only a small number of neuropathic processes affect both autonomic and somatic nerves such as diabetes, amyloidosis, acquired inflammatory demyelinating polyneuropathies, paraneoplastic syndromes, 


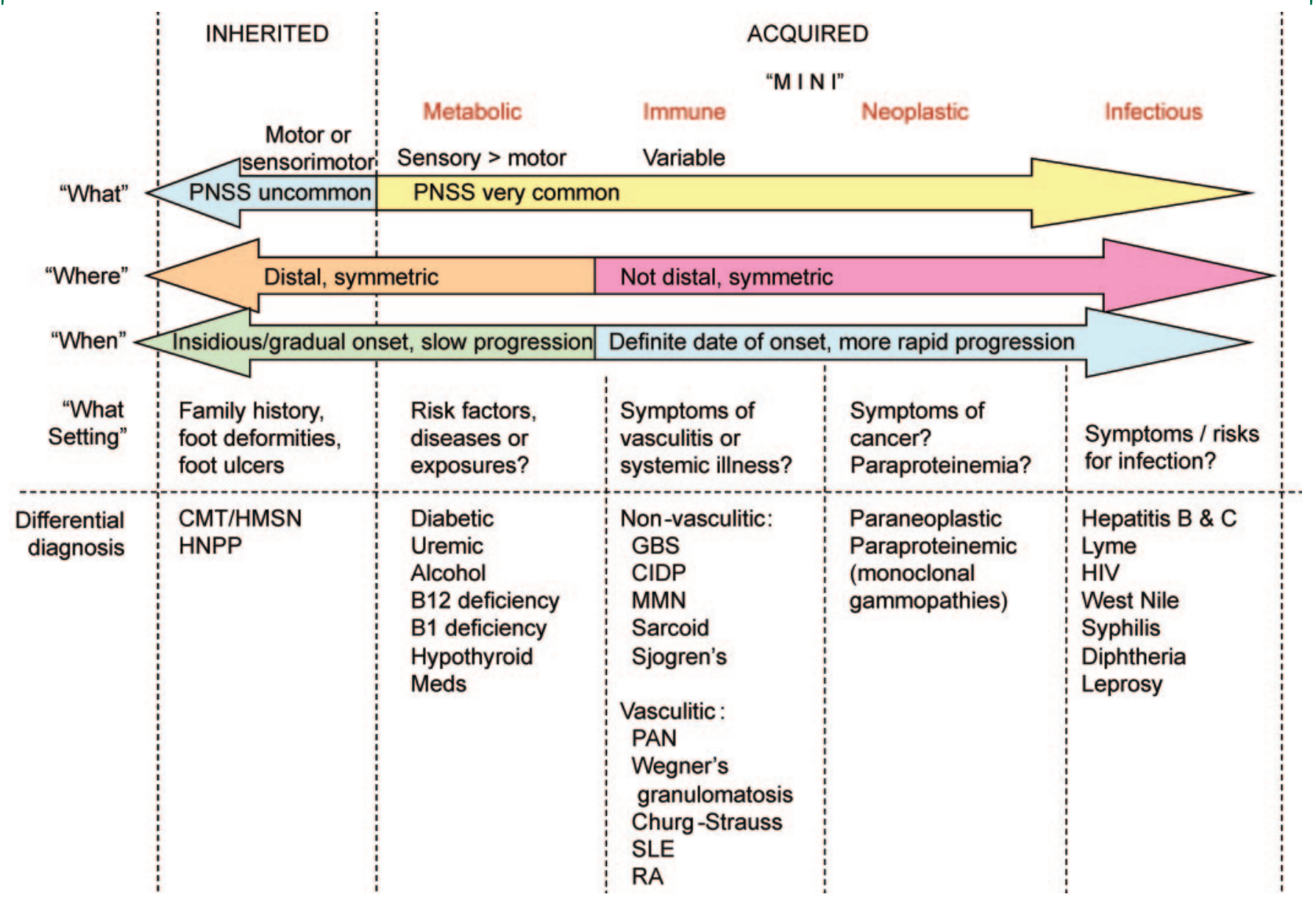

A suggested construct for the approach to neuropathy, using the "what, where, when, and what setting" approach to characterizing the neuropathy and placing the neuropathy into a presumed etiologic category. PNSS = positive neuropathic sensory symptoms; CMT = Charcot-Marie-Tooth; HMSN = hereditary motor and sensory neuropathies; HNPP = hereditary neuropathy with liability to pressure palsy; GBS = Guillain-Barré syndrome; CIDP = chronic inflammatory demyelinating polyradiculoneuropathy; $\mathrm{MMN}=$ multifocal motor neuropathy; PAN = polyarteritis nodosa; $\mathrm{SLE}=$ systemic lupus erythematosus; RA = rheumatoid arthritis. Modified and reprinted with permission from Mauermann ML, Burns TM. The evaluation of acquired neuropathies. Semin Neurol 2008;28:133-151. ${ }^{6}$

Sjögren syndrome, porphyria, HIV, and hereditary sensory and autonomic neuropathies.

Where? Next decide if the distribution is lengthdependent and symmetric or not. Length-dependent neuropathies manifest first in the feet and are symmetric. Non-length-dependent neuropathies may be asymmetric, focal, or multifocal. The etiology of length-dependent neuropathies is usually inherited, metabolic/toxic, or idiopathic, whereas a neuropathy that is not lengthdependent is often caused by an immune-mediated or infectious process. Some examples of non-lengthdependent neuropathies are polyradiculoneuropathies (e.g., acquired inflammatory demyelinating polyneuropathies), plexopathies (often inflammatory), sensory polyganglionopathies (e.g., paraneoplastic subacute sensory neuronopathy caused by small cell lung cancer), and mononeuritis multiplex (caused by vasculitis). At times it may be difficult to determine whether the neuropathy is symmetric or asymmetric due to clinical pro- gression, so it is best to ask the patient to describe the location of onset of symptoms followed by the distribution of subsequent progression.

When? The onset of the neuropathy should be classified. The neuropathies that are acute or subacute in onset are more typical of an immune-mediated or infectious etiology. As patients have difficulty recalling the course, it is helpful to determine whether the symptoms had a definite date of onset. A less-exact date of onset suggests a gradual or insidious onset, indicative of inherited, idiopathic, or toxic/metabolic etiologies. The tempo following symptom onset is also an important consideration. Symptom onset and tempo often correlate in a predictable manner, owing largely to the underlying mechanism. ${ }^{6}$

What setting? The neuropathy has to be considered within the context of the patient's unique clinical circumstances. This is aided by determining what is 
relevant in the patient's medical history, medication list, social history, family history, and the review of systems. An understanding of the significance of these clinical factors requires knowledge of the risk factors of neuropathy and knowledge of the clinical features of the diseases that may be risk factors for neuropathy. For example, unexplained weight loss raises concern for immune or neoplastic causes such as vasculitis, lymphoma, diabetic and nondiabetic lumbosacral radiculoplexus neuropathy, or a paraneoplastic autoimmune process. The clinician should always consider the most common causes of neuropathy such as diabetes, alcohol use, or inherited etiologies. Family members should be examined whenever possible. It is important for the neurologist to obtain an accurate alcohol consumption history and smoking history in a nonthreatening, nonjudgmental manner. Alcoholism is a very common problem and neuropathies due to alcohol use or secondary nutritional deficiencies can occur, so appropriate effort must be spent during the clinic visit investigating this possibility. ${ }^{6,8}$

Clinical neurophysiologic assessment. Characterization using electrodiagnostic testing (EDX) is usually helpful in confirming the presence of a neuropathy and to further define motor and sensory involvement. EDX testing can be normal in small fiber neuropathies, very distal length-dependent neuropathies, and early neuropathic processes, particularly within the first few days. Normal EDX testing can also point to another etiology for the patient's complaints, for example a myelopathy. EDX aids in determining if the neuropathy is primarily axonal or demyelinating. EDX can also help with defining symmetry, length-dependence, and subclinical involvement and provide baseline parameters in case future EDX in needed to monitor the patient's course. Autonomic function may be assessed through autonomic reflex testing or other provocative testing, such as a thermoregulatory sweat test, which specifically assess the autonomic nervous system.

AVOID THE OY-STERS There are three important tips to keep in mind when evaluating peripheral neuropathies.

1. Do not let your sensory examination mislead you. First, your patient almost certainly has sensory involvement if he or she complains of sensory symptoms, regardless of what you find on examination. It is well known that symptoms of sensory involvement are more sensitive for detecting sensory nerve dysfunction than signs on examination. For example, $\sim 75 \%$ of patients with acute inflammatory neuropathies (i.e., Guillain-Barré syndrome) will complain of sensory symptoms at hospital admission but only $\sim 40 \%$ will have confirmatory findings on examination. ${ }^{9}$ Do not let that dissuade you from timely diagnosis and treatment. The second point about the sensory examination is that it is frequently misgraded as abnormal in the unaffected elderly patient and conversely misgraded as normal in the affected young patient. Age (and to a lesser extent height and other anthropomorphic features) must be factored into the judgment of whether sensory testing is normal or abnormal. Also remember that myelopathies can mimic peripheral neuropathy, particularly acute-onset neuropathies such as acute inflammatory neuropathies. Myelopathies often have more profound proprioceptive loss compared to vibration but this is not absolute and the physician should have a low threshold for obtaining imaging of the spinal cord in cases of possible myelopathy. The presence of bowel and bladder impairment can be a helpful differentiator as it strongly favors a myelopathy as the cause.

2. Not all asymmetric neuropathies are acquired. While most inherited neuropathies cause symmetric symptoms and impairments, hereditary neuropathy with liability to pressure palsies (HNPP) often presents with multiple compressive mononeuropathies (e.g., ulnar, median, and peroneal nerves). Thus, HNPP is a relatively common neuropathy that does not conform well to our paradigm. However, family history, foot deformity, subtle details about the history, and the specific EDX characteristics of HNPP usually provide enough clues to prompt consideration and eventual diagnosis. Electrodiagnostic studies demonstrate multiple mononeuropathies with focal conduction blocks as well as generalized prolongation of distal latencies.

3. If it does not fit, acquit. If the characterization of a particular neuropathy does not fit well with the clinical context, for example the medical history or an associated laboratory abnormality, one needs to look elsewhere. Conditions such as diabetes mellitus, hypothyroidism, and renal insufficiency are common in the general population and are most often associated with a length-dependent sensorimotor neuropathy without other systemic features. However, just because these conditions are common does not mean they are always the cause of the neuropathy. Furthermore, these patients are not immune to the development of other acquired neuropathies such as Guillain-Barré syndrome, chronic inflammatory demyelinating polyneuropathy, amyloidosis, or B12 deficiency. For example, approximately $2 \%$ of patients with type 1 diabetes and approximately $6 \%$ of patients with type 2 diabetes have other causes of neuropathy. ${ }^{10}$

\section{REFERENCES}

1. Martyn CN, Hughes RA. Epidemiology of peripheral neuropathy. J Neurol Neurosurg Psychiatry 1997;62:310-318. 
2. England JD, Asbury AK. Peripheral neuropathy. Lancet 2004;363:2151-2161.

3. Dyck PJ, Dyck PJ, Grant IA, Fealey RD. Ten steps in characterizing and diagnosing patients with peripheral neuropathy. Neurology 1996;47:10-17.

4. Dyck PJ, Oviatt KF, Lambert EH. Intensive evaluation of referred unclassified neuropathies yields improved diagnosis. Ann Neurol 1981;10:222-226.

5. Lubec D, Mullbacher W, Finsterer J, Mamoli B. Diagnostic work-up in peripheral neuropathy: an analysis of 171 cases. Postgrad Med J 1999;75:723-727.

6. Mauermann ML, Burns TM. The evaluation of chronic axonal polyneuropathies. Semin Neurol 2008;28:133-151.
7. Bowen JL. Educational strategies to promote clinical diagnostic reasoning. N Engl J Med 2006;355:22172225.

8. Monforte R, Estruch R, Valls-Sole J, Nicolas J, Villalta J, Urbano-Marquez A. Autonomic and peripheral neuropathies in patients with chronic alcoholism: a doserelated toxic effect of alcohol. Arch Neurol 1995;52: $45-51$.

9. Ropper AH. The Guillain-Barré syndrome. N Engl J Med 1992;326:1130-1136.

10. Dyck P. In: Horizons in Mayo Neurology and Neurosurgery: Perspectives on 100 Years and Future Trends. Rochester, MN: 2006. 


\title{
Neurology
}

\author{
Pearls and Oy-sters: Evaluation of peripheral neuropathies \\ Michelle L. Mauermann and Ted M. Burns \\ Neurology 2009;72; $28-\mathrm{e} 31$ \\ DOI 10.1212/01.wnl.0000342135.27500.df
}

This information is current as of February 9, 2009

\author{
Updated Information \& \\ Services \\ Supplementary Material \\ References \\ Subspecialty Collections \\ Permissions \& Licensing \\ Reprints
}

including high resolution figures, can be found at: http://n.neurology.org/content/72/6/e28.full

Supplementary material can be found at: http://n.neurology.org/content/suppl/2009/02/08/72.6.e28.DC1

This article cites 9 articles, 3 of which you can access for free at: http://n.neurology.org/content/72/6/e28.full\#ref-list-1

This article, along with others on similar topics, appears in the following collection(s):

Peripheral neuropathy

http://n.neurology.org/cgi/collection/peripheral_neuropathy

Information about reproducing this article in parts (figures,tables) or in its entirety can be found online at:

http://www.neurology.org/about/about_the_journal\#permissions

Information about ordering reprints can be found online:

http://n.neurology.org/subscribers/advertise

Neurology ${ }^{\circledR}$ is the official journal of the American Academy of Neurology. Published continuously since 1951, it is now a weekly with 48 issues per year. Copyright . All rights reserved. Print ISSN: 0028-3878. Online ISSN: 1526-632X.

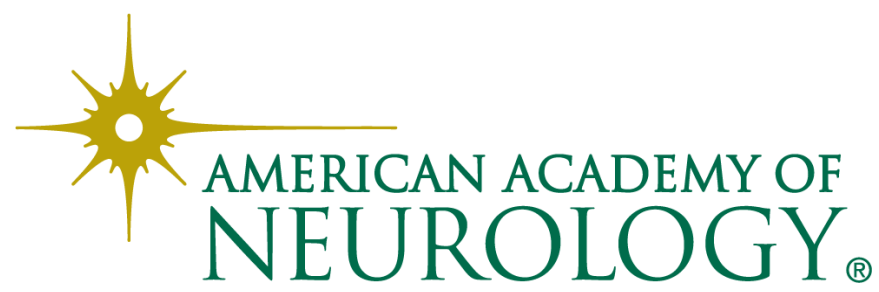

